\title{
ON THE PROBLEM OF CONSTRUCTING ROUTES, PART II: METHODOLOGY AND NUMERICAL EXAMPLE
}

\author{
TRANSLATED AND EDITED BY ILYA GERTSBAKH AND TAO LIU
}

\author{
V.K. Linis, M.S. Maksim \\ E-mail address: sarma.line@gmail.com
}

\begin{abstract}
We consider a periodic aviation schedule which is a finite collection of flights with given arrival/departure times. For this schedule, we solve the problem of finding the minimal number of aircrafts needed to carry out the flights. The crucial role in solving this problem is played by so-called deficit function (DFs) defined as the difference between departures and arrivals on interval [0,t] for each terminal.
\end{abstract}

Keywords: deficit function, optimal chain decomposition, minimal fleet size, centre problem

\section{Problem Description}

Let us assume that each local civil aviation company (LCAC) has a schedule consisting of a list of flights categorized by destinations. This list contains the type of the aircrafts, the frequency of carrying out the flights for each particular destination, and the recommended departure time $t_{0}$ for each particular flight.

When the LCAC is composing the schedule, the main problem is to make sure that all flights in the project could be carried out by a minimal number of aircrafts. This will guarantee the maximal number of flight hours per each operating aircraft. The aircraft which remain "in spare" could be used later for servicing new routes. In practice, it is always possible to nominate for each flight not only its best desirable departure time $t_{0}$, but also a tolerance interval $\left[t_{0}-\Delta \mathrm{t}, t_{0}+\Delta \mathrm{t}\right]$ within which the flight can depart without causing economic damage. It turns out that a small shifting of the departure times within their tolerances allows to minimize the number of aircrafts needed to carry out the schedule. The problem can be descripted as:

Given the preliminary timetable (schedule) for one-type aircraft together with the desirable departure times and their tolerances, work out the final version of the schedule that would provide (1) carrying out all flights within their given tolerances and (2) the minimal possible number of aircrafts in operation involved.

In addition, for each "physical" aircraft in the schedule, there should be determined the so-called turnover route, i.e., the periodic sequence of flights performed by this aircraft, providing periodic aircraft return to its main (base) airport.

\section{Minimal Number of Aircrafts Required to Perform the Planned Schedule}

\subsection{Notations}

The schedule consists of a set of flights numbered from 1 to $R$. Each flight has a departure and an arrival airport. The airports are denoted by the letters $a, b, c, \ldots$ Let $A$ be the set of all airports. Each flight is denoted by the following four-tuple:

$\left(a_{d}^{(r)}, b_{a}^{(r)}, t_{d}^{(r)}, l^{(r)}\right)$,

where $a, b \in A$ are airports, indices $d$ and $a$ denote the departure and arrival, respectively, $t_{d}$ is the departure time, $l$ is the flight duration and $r$ is the flight number.

It is assumed that all flights must be carried out during a fixed time interval $T$, where this interval can be $24 \mathrm{hrs}$, or week or a month. We assume that the following conditions are fulfilled:

$0<t_{d}^{(r)}<T, l^{(r)}<T, r=1,2, \ldots, R$.

The arrival time of the $r$-th flight is given by 


$$
\begin{aligned}
& t_{a}^{(r)}=t_{d}^{(r)}+l^{(r)}, \text { if } t_{a}^{(r)}<T, \text { and } \\
& t_{a}^{(r)}=t_{d}^{(r)}+l^{(r)}-T, \text { if } t_{a}^{(r)} \geq T .
\end{aligned}
$$

Further, we will denote the $r$-th flight by

$$
\gamma(r)=\left(a_{d}^{(r)}, b_{a}^{(r)}, t_{d}^{(r)}, l^{(r)}\right) .
$$

It will be assumed that there are no coincidences among the set of arrival and departure times and no one of them is equal to 0 or $T$.

The upper part of Figure 1 presents a picture of a schedule. The flight $r$ is shown by a segment with the beginning at time $t_{d}^{(r)}$ and the end at $t_{a}^{(r)}$.
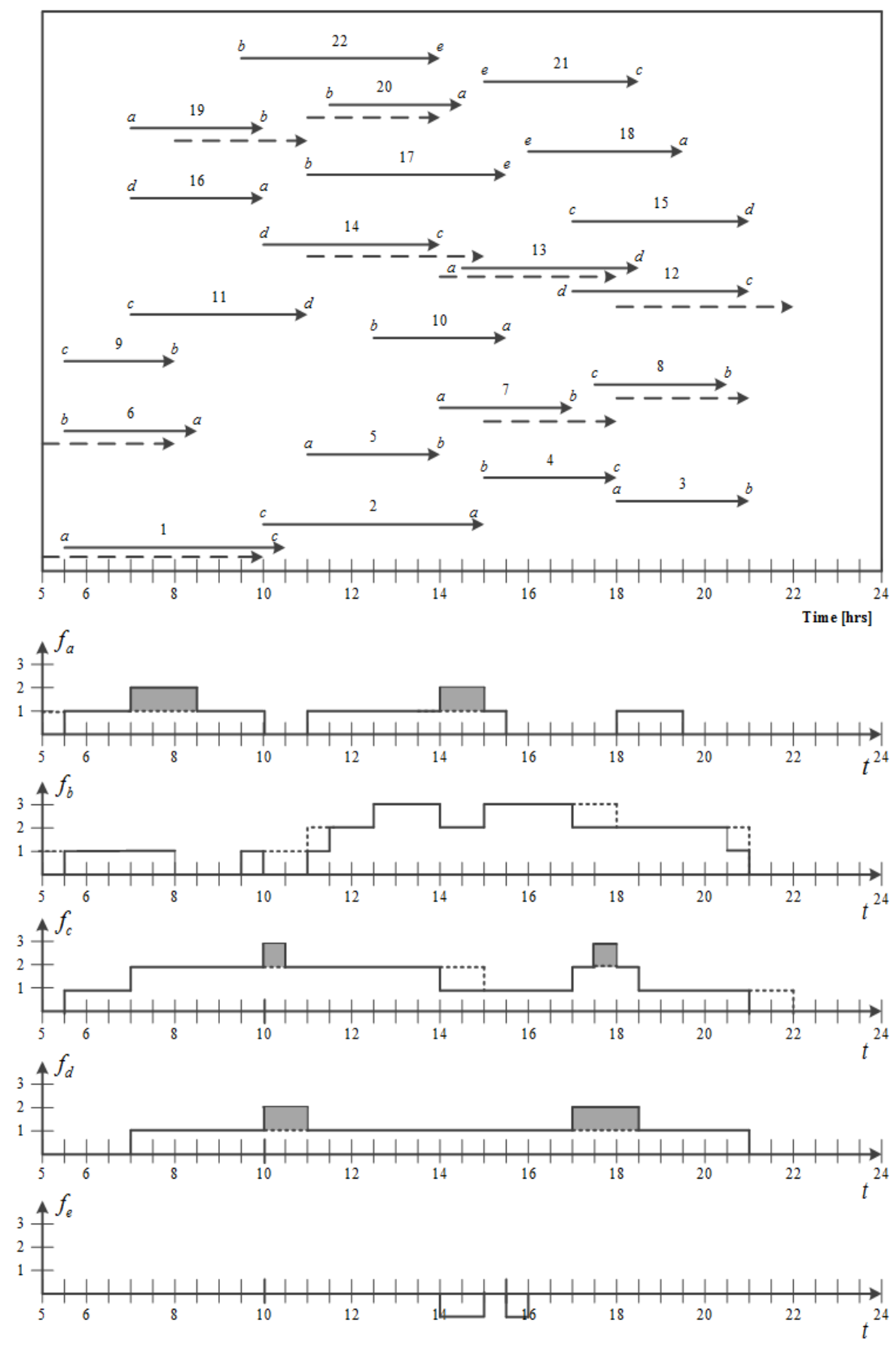

Figure 1. Upper part: the original schedule (before shifting flight departure times); lower part: the corresponding characteristic functions 


\subsection{Fleet size formula}

Let us find out what the minimal number of aircrafts needed is so as to carry out the given set of flights. To carry out the $r$-th flight, departing from $v$ there should be an aircraft available at airport $v$ at the instant $t_{d}^{(r)}$. After the departure takes place, the number of aircrafts in $v$ will decrease by one. This number will increase by one at the instant of any flight arrival to $v$.

Let us construct for each airport $v$ its stepwise (or characteristic) function $f_{v}(t), v \in A$, $0 \leq t \leq T$ which is defined for each $t$ as the difference between the number of departures from $v$ and the number of arrivals to $v$ on the interval $[0, t]$. See Fig. 1.

Suppose that at the moment $t=0$ there are $C_{v}$ aircrafts on the ground in $v$. Then at time $t$ there will be $C_{v}-f_{v}(t)$ aircrafts in $v$. Obviously, that in order to carry out all flights departing from $v$ it is necessary and sufficient that the following condition should be fulfilled:

$C_{v}-f_{v}(t) \geq 0,0 \leq t \leq T$

This will be satisfied if we put $C_{v}=\max _{0 \leq t \leq T} f_{v}(t)$. To make possible all other flights, it is necessary and sufficient to fulfil the condition (6) for all $v \in A$. In addition, at $t=0$ some number $D$ of aircrafts are in the air. Taking into account that at any time the number of aircrafts on the ground and in the air is the same, we arrive at the following statement:

The minimal number of aircrafts needed for carrying out the schedule $\gamma(1), \ldots, \gamma(\mathrm{R})$ equals

$Q=\sum_{v \in A} \max _{0 \leq t \leq T} f_{v}(t)+D$

\section{Constructing Closed Flight Chains (Cyclic Diagrams)}

\subsection{Assumptions}

It is assumed that the collection $\gamma(1), \ldots, \gamma(\mathrm{R})$ has the natural "balance" property that the number of arrivals to each airport is equal to the number of departures from it. It means that there is no aircraft dropout from the schedule.

\subsection{Minimal Complete Chain System}

Let us call a chain the following ordered sequence of flights $z=<\gamma\left(l_{1}\right), \gamma\left(l_{2}\right), \ldots, \gamma\left(l_{n}\right)$, having the following closure property:

$b_{a}^{l_{n}} \equiv a_{d}^{l_{1}}, b_{a}^{l_{1}} \equiv a_{d}^{l_{2}}, \ldots, b_{a}^{l_{n-1}} \equiv a_{d}^{l_{n}}$.

Often, a chain is called cyclic diagram.

The property of a chain is that its flights $\gamma(1), \ldots, \gamma(n)$ can be performed by the same "physical" aircraft. This aircraft will periodically circulate along this chain. The whole schedule $\gamma(1), \ldots, \gamma(n)$ can be decomposed into nonintersecting subsets, each of which itself is a chain. This can be easily proved by using the conditions stated in subsection 3.1. We will call a system (a set) of chains complete if each of the flights $\gamma(1), \ldots, \gamma(n)$ is present in one and only one chain.

Let us consider a chain $z$ and denote by $L_{z}$ the number of $T$ periods, which a single aircraft needs to carry out the whole cycle along this chain. Let us call $L_{z}$ the weight of the chain $z$. If $L_{z}$ equals integer $m$, and all flights of this chain are carried out during one period [0, $T$ ], then $m$ "physical" aircrafts should circulate along this chain. These aircrafts should perform the same flight $m$ times, with time interval $T$. Therefore, the sum of all the weights of all chains $L=\sum L_{Z}$ is equal to the total number of aircrafts which carry out the schedule. This system (set) of chains is called minimal, if

$L=Q$. 


\subsection{Algorithm for Constructing the Minimal Complete Chain System}

Let us describe the algorithm for constructing the minimal complete chain system (MCCS). Take any flight $\gamma\left(l_{1}\right)$ arriving at airport $b$ at time $t_{1}$ and join to it the nearest flight $\gamma\left(l_{2}\right)$ departing from $b$ at time $t_{d}$. The "nearest" means the following: either it is the flight whose departure from $b$ is in the interval $\left[t_{1}, T\right]$, or, if such does not exist, then it is the first departure from $b$ after $t=0$ on the next period. Similarly, we join the flight $\gamma\left(l_{3}\right)$ to $\gamma\left(l_{2}\right)$, and so on, until the chain closes, i.e. the closest departure after arrival of $\gamma\left(l_{n}\right)$ will be the departure by $\gamma\left(l_{1}\right)$.

Finally, all flights of one chain are removed from the schedule, and the similar procedure is repeated for the remaining set of flights, until the whole schedule is exhausted.

\subsection{Extension of the Minimal Complete Chain System}

It follows from subsection 3.3 that constructing the MCCS can be made in several ways. Suppose there are two MCCS's, containing $M_{1}$ and $M_{2}$ chains, respectively, and $M_{1}<M_{2}$ It is natural to prefer the second MCCS with the largest $M$-value because this MCCS will consist of chains which, on the average, are shorter.

\section{Constructing Closed Chains to Visit the Base Airports}

Let $A_{1} \subset A$ be the set of airports equipped with special facilities. We call them base airports. We would like to construct a system of chains in such a way that every chain would visit an airport belonging to $A_{1}$. More specifically, we want to design an MCCS in which every chain visits a base airport. Let us present without proof the condition guaranteeing such property.

Let us find for each $a \in A$, the time segments which are the "hollow-zones" between two adjacent maximums of the characteristic function $f_{a}(t)$. Let these zones be numbered in cyclic order as $a_{1}, a_{2}, \ldots$, $a_{m}$. Suppose that there is a departure of a flight from zone $a_{1}$ which has the arrival in some hollow zone $b_{j}$ of airport $b$. Then we say that there is a transition from $a_{1}$ to $b_{j}$. Let $E_{1}$ be the set of all hollow-zones into which there is a transition from $a_{1}$. Let us complement it by the set $E_{2}$ of all hollow-zones into which the transition from $E_{1}$ is possible, and so on. Eventually, we either will exhaust all hollow zones, or not. In the last case, we repeat the similar procedure for the remaining hollow- zones. This will result in the partition of the whole set of hollow-zones into sets $B_{1}, B_{2}, \ldots, B_{s}$ which have the following property: the hollow-zones within each $B j$ communicate with each other, while the hollow-zones from different $B_{j}$-s do not. Now we formulate without proof the following claim:

In order to construct a MCCS with each chain visiting a base, it is necessary and sufficient that each set $B j, j=1, \ldots, s$ would contain a hollow-zone belonging to a base airport.

\section{Algorithm for Solving the Routing Problem}

The theoretical considerations developed in the previous sections allow to propose an algorithm for solving the problem of route construction. This solution has several stages.

a. For each type of aircraft, LCAC composes a preliminary list of daily flights $\mathrm{L}^{*}=(\gamma *(1), \ldots, \gamma *(R *))$ which have to be carried out in a period [0,T]. Typically, this period is taken as 7 days (one week).

b. $L *$ is complemented by non-daily flights, if there are such. These flights are approximately uniformly positioned over the week period.

c. For each flight, the desirable departure time is nominated. If the flight has several legs, these times take into account the minimal time needed to stay on the ground between the arrival and the departure.

d. The "ideal" departure/arrival times are coordinated with the operation time for each airport. These times are chosen also by taking into account the passenger demand and passenger convenience. In addition, each departure time $t_{0}$ is supplied with a tolerance $\Delta t$, so that the departure can be within the interval $\left[t_{0}-\Delta t, t_{0}+\Delta t\right]$.

e. After completing the stages b-e, LCAC arrives at the complemented list of flights $L=(\gamma(1), \ldots, \gamma(R))$ which contains all daily and non-daily flights, together with their arrival/departure times. This list is 
used to determine the characteristic functions $f_{a}(t)$ for each $a \in A$. Finally, the minimal number of aircrafts $Q$ to carry out the schedule is determined.

f. The departure times of certain flights are changed within their tolerances in order to lower the maxima of the characteristic functions. As it follows from Section 2, this guarantees the decrease of $Q$. It is essential that repositioning of flight departure times involves only the flights which are located in the neighbourhood of $f_{a}(t)$ maxima.

g. After the decrease of $Q$ by one unit has been achieved, the resulting MCCS is checked whether it satisfies the base principle (see section 4 ). If yes, the stage $\mathbf{f}$ can be repeated.

h. Using the algorithm described in Section 3, the MCCSs are constructed and their best version, according to the criterion of subsection $\mathbf{3 . 4}$, is selected.

\section{An Example}

Let us consider an example that illustrates in somewhat simplified form of the algorithm described in Section 5.

The schedule is presented on the upper part of Fig. 1. It contains 22 daily flights shown by line segments numbered from 1 to 22 . All flights have their arrival and departure times between 5 am and 24 $(12 \mathrm{pm})$. The arrival/departure airports are shown by letters at the end points of the segments. The length of each segment represents the flight time plus the necessary time on the ground to prepare the aircraft to the next flight and passenger boarding. The right end of each segment has an arrow indicating the instant when the next departure can be carried out.

All tolerances of departure times are $\pm 2 \mathrm{hrs}$. The earliest departure time is 5 am and the latest arrival time is $10 \mathrm{pm}$.

The lower part of Fig. 1 shows the characteristic functions for all five airports $a, b, d, c, e$ in the schedule. It is seen that $D=0, \sum_{v \in A} \max _{0<t<24} f_{v}(t)=10$.

Now let us modify the schedule in order to lower the maximum areas of the characteristic functions. First we take $f_{\mathcal{C}}(t)$ that haves the narrowest maximum areas. To "erase" the left maximum, we shift the first flight to the left by half an hour (denote it as $N o .1 \Leftarrow 0.5 \mathrm{hr}$ ). The erased left maximum is shown by grey colour. To erase the second maximum, we carry out the shifting of No.8 $\Rightarrow 0.5 \mathrm{hr}$. Note that doing these shiftings results in changes of $f_{a}(t)$ and $f_{b}(t)$ because the shifted flights had arrival/departure from these airports. As the result, $f_{a}(t)$ increases in the interval $[5: 00,5: 30]$ and $f b(t)$ increases in the interval [20:30,21:00]. We see that this does not affect the maxima of $f_{a}$ and $f b$.

To erase the left maximum area of $f_{d}(t)$ we shift $N o .14 \Rightarrow 1 h r$, and to erase the right maximum area we need three shiftings: No. $12 \Rightarrow 1 \mathrm{hr}$, No. $13 \Leftarrow 0.5 \mathrm{hr}$ and No.20 $\Leftarrow 0.5 \mathrm{hr}$. These shiftings will cause changes of $f_{a}, f_{b}, f_{c}$ shown by dotted colour. The maximum zones of $f_{a}(t)$ are erased by the shiftings of No. $19 \Rightarrow 1 h r$, No. $6 \Leftarrow 0.5 h r$ and No. $\Rightarrow 1 h r$.

The new positions of the shifted flights are shown by dotted lines. After carrying out all the above shiftings we have achieved the decrease of $Q$ by 3 units and now $Q=7$. This process can be continued further, and we omit the details. Fig. 2 demonstrates the final result. Now $f_{e}(t)=0$ because the arrival times at $e$ coincide with the corresponding departure times from $e$. The value of $Q$ is now equals to 5 , a considerable decrease comparing to its initial value of 10 . the chain:

Now let us construct the MCCS. Start with flight No.19. Joining the nearest departure, we obtain

No. $19 \rightarrow$ No. $17 \rightarrow$ No. $18 \rightarrow$ No. $3 \rightarrow$ No.6.

with a weight of 1 . See Fig. 2.

Starting with flight 5 , we will obtain the chain:

No. $5 \rightarrow$ No. $20 \rightarrow$ No. $13 \rightarrow$ No. $12 \rightarrow$ No. $9 \rightarrow$ No. $22 \rightarrow$ No. $21 \rightarrow$ No. $15 \rightarrow$ No. 16.

Its weight is equal to 2 . The third chain, also with a weight of 2 , is the following:

No. $1 \rightarrow$ No. $2 \rightarrow$ No. $7 \rightarrow$ No. $4 \rightarrow$ No. $11 \rightarrow$ No. $14 \rightarrow$ No. $8 \rightarrow$ No. 10.

It can be seen now that each of the above chains visits airport $a$. If $a$ is a base, then we have obtained an MCCS in which all aircraft will visit the base airport. 

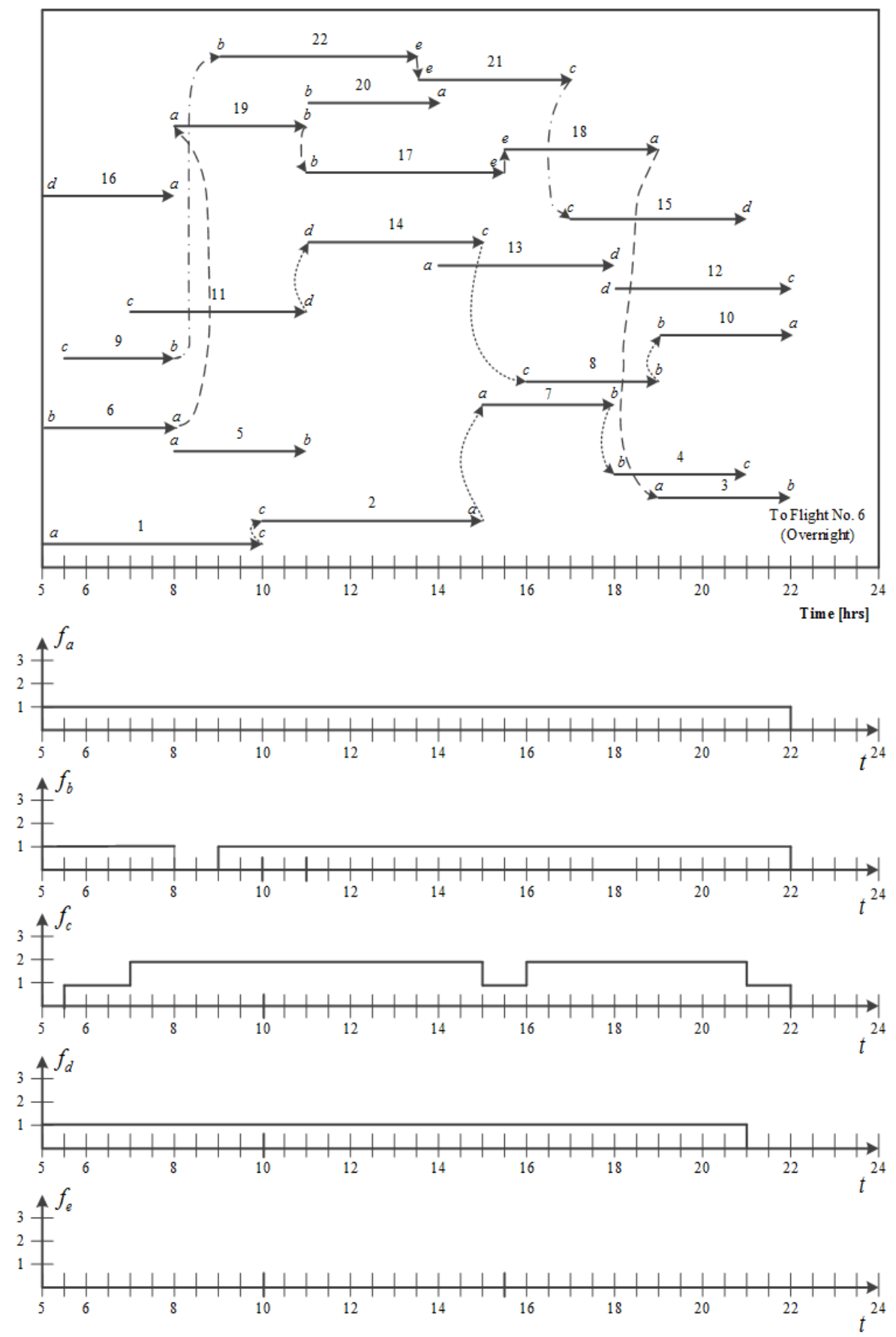

Figure 2. Upper part: final position of the flights after shifting departure times; lower part: the corresponding characteristic functions

\section{Concluding Remark}

Let us note that solving the routing problem can be made at various levels. That is, it can be solved for a single local airline company or simultaneously for several local companies exploiting identical (mutually exchangeable) aircrafts. Certainly, the efficiency of the described methodology of this work will increase with the increase of the number of flights and number aircrafts employed. 